\title{
Diagnóstico não Invasivo da Anemia Fetal pela Medida do Pico de Velocidade Sistólica na Dopplervelocimetria da Artéria Cerebral Média
}

\author{
Noninvasive Fetal Anemia Diagnosis by Middle Cerebral Artery \\ Peak Systolic Velocity Waveform Measurement \\ Marcos Roberto Taveira, Antônio Carlos Vieira Cabral, Henrique Vítor Leite, \\ Isabela Gomes de Melo, Ana Paula Brum de Miranda Lopes
}

\begin{abstract}
RESUM0
Objetivo: avaliar se existe associação entre a medida do pico de velocidade sistólica (PVS) na dopplervelocimetria da artéria cerebral média (ACM) e a concentração de hemoglobina fetal e determinar a sua capacidade diagnóstica.

Métodos: entre janeiro de 2000 e maio de 2003, 44 gestantes isoimunizadas foram submetidas a transfusão intra-uterina. Realizou-se estudo dopplervelocimétrico da ACM antes de cada transfusão fetal, sempre com intervalo inferior a 3 horas, antecedendo o procedimento. O PVS da ACM foi considerado alterado quando seu valor era superior a 1,5 múltiplo da mediana para a respectiva idade gestacional. A concentração de hemoglobina do cordão foi aferida antes de se iniciar a infusão de sangue, realizada no Hemocue ${ }^{\circledast}$ (B-Hemoglobin Photometer Hemocue AB; Angelholm, Sweden). O estudo estatístico foi feito pelo teste do $\chi^{2}$ e também foram calculados os valores de sensibilidade, especificidade, valores preditivos positivo e negativo.

Resultados: foram realizados 83 procedimentos, sendo que em 33 a hemoglobina fetal era inferior a $10,0 \mathrm{~g} / \mathrm{dL}$. Houve associação significativa entre as variáveis estudadas, $p<0,001$. Fetos com hemoglobina menor ou igual a $10 \mathrm{~g} / \mathrm{dL}$ apresentaram aumento significativo na medida do PVS na ACM, com valores superiores a 1,5 múltiplo da mediana. O PVS na ACM apresentou sensibilidade de $75,7 \%$ para a detecção de hemoglobina do cordão menor ou igual a $10 \mathrm{~g} / \mathrm{dL}$.

Conclusão: o pico de velocidade sistólica na artéria cerebral média pode ser utilizado como método complementar no diagnóstico da anemia fetal.
\end{abstract}

PALAVRAS-CHAVE: Gravidez de alto risco. Isoimunização ao fator Rh. Anemia fetal.

\section{Introdução}

O progressivo desenvolvimento da propedêutica obstétrica tem propiciado o surgimento de técnicas não invasivas para avaliação das condições fetais. Uma situação clínica em que se busque melhor aplicação dos métodos não invasivos é quando há risco para anemia fetal. Isso pode significar redução acentuada no número de procedimentos invasivos, reservando-os apenas para os

Centro de Medicina Fetal do Hospital das Clínicas - UFMG Correspondência:

Marcos Roberto Taveira

Rua dos Otoni 909/sala 2108 - Bairro Santa Efigênia

30150-270 - Belo Horizonte - MG casos mais graves, nos quais há indicação para tratamento intra-uterino.

Em nosso meio, a isoimunização materna pelo fator Rh é a causa mais freqüente de hemólise fetal. O diagnóstico do grau de anemia fetal pode ser determinado por meio da amniocentese, quando se realiza a espectrofotometria do líquido amniótico, ou pela dosagem da hemoglobina no sangue obtido por cordocentese. Os métodos invasivos citados apresentam riscos para gestante e para o feto, além de que devem ser repetidos com alguma freqüência para o acompanhamento dos valores da hemoglobina fetal, no evolver da gestação $0^{1,2}$.

Dos métodos não invasivos estudados para 
detecção da anemia fetal, destaca-se a dopplervelocimetria. Além de ser seguro e permitir o estudo da hemodinâmica fetal, auxilia na detecção dos mecanismos da adaptação do concepto frente à anemia ${ }^{3}$.

A dopplervelocimetria arterial já foi pesquisada com a finalidade de identificar marcadores da anemia fetal, tendo-se conseguido demonstrar que fetos anêmicos apresentam estado hiperdinâmico de fluxo evidenciado pelo aumento nas velocidades em alguns vasos, tais como, artéria esplênica, aorta torácica descendente e principalmente artéria cerebral média (ACM). Estes achados indicam que o feto anêmico aumenta o débito cardíaco na tentativa de manter adequado aporte de oxigênio aos seus órgãos e tecidos ${ }^{4-7}$.

$\mathrm{O}$ feto anêmico apresenta aumento na velocidade média de fluxo detectado no exame dopplervelocimétrico da ACM, que é inversamente proporcional ao nivel de hemoglobina fetal ${ }^{7}$.

Nos últimos anos, alguns autores observaram que há correlação entre o aumento do pico de velocidade sistólica (PVS) na ACM, e o grau de anemia fetal. A sensibilidade deste parâmetro variou entre 91 e 100\%, mesmo nos casos com anemia grave, com hemoglobina fetal inferior a $6 \mathrm{~g} / \mathrm{dL}$, e com especificidade de $100 \%$, para uma taxa de falso-positivo de $18 \%$. O aumento do PVS, com valores maiores do que 1,5 múltiplo da mediana, para a respectiva idade gestacional, indica a existência de aumento compensatório no débito cardíaco em resposta à redução na viscosidade sangüínea ${ }^{6,8}$.

Fetos anêmicos apresentam também aumento no ângulo de inclinação na medida do PVS na dopplervelocimetria da ACM, sendo este aumento proporcional ao grau de anemia fetal, indicando também aumento compensatório no débito cardíaco. Imediatamente após as transfusões intrauterinas ocorre redução significativa no PVS, validado pela dopplervelocimetria da ACM, indicando haver redução no débito cardíaco fetal, podendo ser indício da correção da anemia. O seu uso no diagnóstico não invasivo das anemias pode evitar a realização das cordocenteses propedêuticas, reservando-as apenas para o tratamento da doença ${ }^{9-11}$.

O PVS na ACM apresentou sensibilidade e valor preditivo positivo de 75 e $60 \%$ e 100 e $47 \%$ para um défict de hemoglobina de -3 e -5 desvios padrão, respectivamente. A amniocentese apresentou sensibilidade e valor preditivo positivo iguais a zero e de 86 e 100\% para os mesmos níveis de anemia fetal. O PVS na ACM e a amniocentese foram igualmente eficientes para a detecção da anemia fetal grave, entretanto, com a dopplervelocimetria, pode-se reduzir o número de procedimentos invasivos realizados ${ }^{12}$.

A medida do PVS na dopplervelocimetria da ACM apresentou sensibilidade de 100\%, valor preditivo positivo de $71 \%$ e uma taxa de falso-positivo de $28 \%$, sendo superior aos métodos convencionais de diagnóstico da anemia fetal. A amniocentese e a cordocentese propedêuticas apresentaram valores de 80, 44 e 56\%, respectivamente, para sensibilidade, valor preditivo positivo e falsopositivo. Atualmente tem sido considerado o melhor método para o diagnóstico não invasivo da anemia fetal, independente de sua causa, desde que realizado por examinadores experientes, para que não ocorram erros na realização do exame ${ }^{13,14}$.

O objetivo deste estudo foi estabelecer a existência de associação entre o aumento no PVS da ACM e a presença de anemia no concepto, avaliando a capacidade diagnóstica do método para a determinação da gravidade do quadro de anemia fetal.

\section{Pacientes e Métodos}

No período de janeiro de 2000 a maio de 2003 foram acompanhadas 44 gestantes isoimunizadas pelo fator Rh no Centro de Medicina Fetal do Hospital das Clínicas da UFMG (CEMEFE). Cada transfusão intra-uterina seguida da coleta do sangue fetal e precedida da dopplervelocimetria da ACM foi considerada um caso, totalizando 83 procedimentos analisados. Desta forma, um mesmo feto pode ter sido incluído no estudo mais de uma vez, de acordo com o número total de procedimentos aos quais tenha sido submetido.

As transfusões intra-uterinas foram indicadas quando havia indícios de concentração de hemoglobina fetal inferior a $10 \mathrm{~g} / \mathrm{dL}$ (espectrofotometria de líquido amniótico nas zonas $1 \mathrm{~A}$ e $1 \mathrm{~B}$, sinais de hidropisia fetal à ultrasonografia e mau passado obstétrico para a isoimunização Rh), com idade gestacional inferior a 34 semanas $^{15}$.

A dopplervelocimetria da ACM, foi efetuada com intervalo máximo de 3 horas antes do momento da cordocentese para determinação da hemoglobina de cordão.

A mensuração da concentração de hemoglobina fetal foi realizada no Hemocue ${ }^{\circledR}(B-H e m o g l o b i n$ Photometer Hemocue AB; Angelholm, Sweden).

O exame dopplervelocimétrico da ACM foi realizado por um único examinador, com a paciente em posição de semi-Fowler, estando o feto em apnéia e na ausência de movimentos corporais. Os exames foram realizados em aparelhos Sonoace 8800 da Medson. Utilizou-se filtro acústico de $100 \mathrm{~Hz}$. O fluxograma foi obtido após a observação de pelo menos cinco ciclos cardíacos consecutivos e uniformes, sendo calculada a média aritmética de três ciclos. A ACM foi insonada pró- 
ximo a sua origem no polígono de Willis, ou então em seu trajeto ao longo do giro da ínsula.

O parâmetro dopplervelocimétrico estudado na ACM desses fetos foi o pico de velocidade sistólica, sempre corrigindo o ângulo entre o transdutor e o vaso insonado para o mais próximo de zero, até um máximo de $60^{\circ}{ }^{6}$.

O sangue fetal foi obtido por punção da veia umbilical durante a realização de cordocentese, guiada pela ultra-sonografia, para avaliar a necessidade da possivel transfusão intra-uterina.

Definiu-se o grau de anemia fetal de acordo com a concentração de hemoglobina fetal, sendo considerados graves os casos com nivel de hemoglobina menor ou igual a $7 \mathrm{~g} / \mathrm{dL}$. Anemia moderada foi definida pela hemoglobina do feto entre $7 \mathrm{e}$ $10 \mathrm{~g} / \mathrm{dL}$ e, anemia leve com hemoglobina entre 10 e $12 \mathrm{~g} / \mathrm{dL}^{1}$.

As pacientes foram orientadas quanto ao estudo e assinaram termo de consentimento prévio para a realização dos procedimentos invasivos e não invasivos. A pesquisa foi aprovada pela Comissão de Ética em Pesquisa do Hospital das Clínicas da UFMG (COEP HC UFMG).

Após obtidos os resultados, realizou-se estudo de associação entre o PVS e a concentração da hemoglobina no cordão. Utilizou-se, para comparação, a curva de normalidade proposta por Mari et al. ${ }^{6}$, sendo considerados alterados os valores do PVS que fossem superiores a 1,5 múltiplo da mediana, para a respectiva idade gestacional ${ }^{6}$.

Para o estudo de associação calculou-se o $\chi^{2}$, considerando significativo um valor de $\mathrm{p}<0,05$, por meio do software Minitab 2000.

Procurou-se avaliar a acuidade do PVS para o diagnóstico não invasivo de anemia fetal, em sua forma moderada e grave, calculando a sensibilidade, especificidade e valores preditivos, por meio do software Epi-Info 2000.

\section{Resultados}

Houve um total de 83 transfusões intrauterinas em 44 fetos, com média de 1,9 procedimento por feto, mínimo de 1 e máximo de 6 procedimentos.

Nos casos acompanhados, a concentração média da hemoglobina no cordão foi de 9,9 g/dL, com mediana de $10,4 \mathrm{~g} / \mathrm{dL}$, valor mínimo de 3,3 g/dL e máximo de 16,4 g/dL. A idade gestacional média foi de 26,7 semanas, com mediana de 28 semanas, mínimo de 20,5 e máximo de 34,4 semanas de gestação.

O valor médio do PVS no Doppler da ACM foi de $53,5 \mathrm{~cm} / \mathrm{s}$, com mediana de $49,1 \mathrm{~cm} / \mathrm{s}$, valor mínimo de 19,7 cm/s e máximo de $137 \mathrm{~cm} / \mathrm{s}$.
O estudo revelou associação significativa entre o PVS na dopplervelocimetria da ACM, com a anemia fetal, $\mathrm{p}<0,001$, demonstrando que fetos com concentração de hemoglobina menor ou igual a $10 \mathrm{~g} / \mathrm{dL}$ apresentaram aumento significativo na medida do PVS na ACM, com valores acima de 1,5 múltiplo da mediana (Tabela 1).

Tabela 1 - Associação entre o pico de velocidade sistólica na artéria cerebral média e concentração de hemoglobina fetal em fetos de gestantes isoimunizadas.

\begin{tabular}{lccc}
\hline & $\mathbf{H b} \leq \mathbf{1 0} \mathbf{~ g / d L}$ & $\mathbf{H b}>\mathbf{1 0} \mathbf{~} / \mathbf{d L}$ & Total \\
\hline PVS alterado & 25 & 11 & 36 \\
PVS normal & 8 & 39 & 47 \\
Total & 33 & 50 & 83 \\
\hline
\end{tabular}

$\chi^{2}=23,3 p<0,001$, teste de Yates, $p<0,001$

Sensibilidade $=75,8 \%$

Especificidade $=78,0 \%$

Valor preditivo positivo $=69,5 \%$

Valor preditivo negativo $=82,4 \%$

$\mathrm{PVS}=$ pico de velocidade sistólica $; \mathrm{Hb}=$ hemoglobina; $\chi^{2}=$ qui-quadrado.

O valor alterado do PVS no Doppler da ACM apresentou, na detecção de hemoglobina fetal menor ou igual a $10 \mathrm{~g} / \mathrm{dL}$, sensibilidade de $75,8 \%$, especificidade igual a $78 \%$, valor preditivo positivo de $69,5 \%$ e valor preditivo negativo de $82,9 \%$ (Tabela 1).

\section{Discussão}

Apesar dos grandes progressos realizados na profilaxia da sensibilização materna pelo fator $\mathrm{Rh}$, ainda são freqüentes os casos de gestantes sensibilizadas, o que provoca a possibilidade de anemia fetal. Nestes casos, a determinação do grau de anemia fetal é ainda realizada por meio de procedimentos invasivos, seja amniocentese ou cordocentese.

A espectrofotometria de líquido amniótico, obtido pela amniocentese, apresenta os mesmos riscos que qualquer procedimento invasivo (infecção, amniorrexe, aumento do grau de sensibilização) ${ }^{1,2}$.

A punção do cordão umbilical, que permite determinar de forma direta o nível de hemoglobina fetal, apresenta o risco de hemorragia fetomaterna em até 50\% dos procedimentos, associando-se ainda com taxas de perdas fetais entre 1,5 e $5 \%$, condicionadas principalmente à experiência do examinador ${ }^{2,16-18}$.

Recentemente, intensificou-se a busca por um método propedêutico não invasivo, com alta sensibilidade para a detecção da anemia fetal, evitando-se assim as complicações potencialmente graves relatadas no uso dos procedimentos invasivos. 
Esse método permitiria individualizar a assistência aos fetos anêmicos, selecionando melhor os casos que se beneficiariam da propedêutica invasiva.

A dopplervelocimetria tem sido estudada com o objetivo de determinar a presença da anemia fetal. Os estudos envolvendo a dopplervelocimetria do compartimento arterial, na pesquisa da anemia fetal, concluíram que fetos anêmicos exibem aumento global nas velocidades de fluxo em resposta à hemólise. Isso caracteriza um estado de fluxo hiperdinâmico secundário à redução da viscosidade sangüínea e à queda do hematócrito, resultando em aumento do débito cardíaco, visando manter constante o aporte de oxigênio aos órgãos e tecidos fetais ${ }^{3,5,6,17,19}$.

Nossos resultados comprovaram haver associação significativa entre o aumento no PVS na ACM e a redução na concentração de hemoglobina fetal. Verificamos que o método apresentou sensibilidade de 75,8\% no diagnóstico da anemia fetal grave e moderada (hemoglobina menor ou igual a $10 \mathrm{~g} / \mathrm{dL}$ ).

Comparando os nossos resultados quanto ao valor da sensibilidade do PVS no diagnóstico de anemia fetal, observamos que são inferiores aos relatados por outros autores, que inicialmente observaram sensibilidade variando entre 91 e $100 \%$, mesmo nos casos graves, com hemoglobina abaixo de $6 \mathrm{~g} / \mathrm{dL}$.

Sabe-se que, em fetos anêmicos, a mudança no hematócrito leva a uma alteração correspondente na viscosidade sangüinea e a uma diminuição na liberação tecidual de oxigênio. O aumento no débito cardíaco e a vasodilatação são os principais mecanismos pelos quais o feto tenta manter o aporte adequado de oxigênio para os diversos órgãos. Entretanto, alguns autores observaram que fetos em estágios mais avançados de anemia poderiam já exibir alterações em sua função cardíaca, sendo, portanto, incapazes de elevar seu débito cardíaco. Portanto, nestes casos pode não ocorrer elevação nas velocidades de fluxo nos diversos leitos vasculares, incluindo a ACM. Fetos gravemente anêmicos, com concentração de hemoglobina menor que $7 \mathrm{~g} / \mathrm{dL}$, podem não exibir um estado hiperdinâmico de fluxo, justificando, desta forma, a diferença em nossos resultados ${ }^{20,21}$.

No grupo estudado, dos 83 casos previamente selecionados, 50 apresentavam hemoglobina fetal maior ou igual a $10 \mathrm{~g} / \mathrm{dL}$. Neste subgrupo, após a coleta do sangue fetal, enquanto se aguardava o resultado da concentração de hemoglobina no Hemocue ${ }^{\circledR}$, era iniciada a infusão de sangue. Imediatamente após a liberação desse resultado (tempo médio de 1 minuto) e confirmado nível de hemoglobina maior que $10 \mathrm{~g} / \mathrm{dL}$, o procedimento era interrompido. Nessas situações o volume má- ximo de sangue transfundido não ultrapassava 10 $\mathrm{mL}$, o que certamente não implicaria riscos adicionais para o feto ${ }^{15}$.

Observamos também que, dos 50 casos que apresentavam concentração de hemoglobina fetal superior a $10 \mathrm{~g} / \mathrm{dL}, 11$ (22\%) apresentaram aumento no PVS na dopplervelocimetria da ACM. Pode-se explicar este achado ou por uma taxa elevada de falso-positivo ou supondo-se que, nos fetos em estágios iniciais do processo anêmico, já poderia existir o aumento compensatório no débito cardíaco fetal. Um estudo posterior, estratificando os fetos por faixas mais estreitas dos níveis de hemoglobina (abaixo de $4 \mathrm{~g} / \mathrm{dL}$; entre 4 e $6 \mathrm{~g} / \mathrm{dL}$, entre 6 e $8 \mathrm{~g} / \mathrm{dL}$, entre 8 e $10 \mathrm{~g} / \mathrm{dL}$ e entre 10 e 12 $\mathrm{g} / \mathrm{dL}$ ), poderia vir a esclarecer esta dúvida.

Diante dos resultados aqui obtidos pode-se concluir que o aumento na medida do PVS no estudo dopplervelocimétrico da ACM associou-se significativamente a nivel de hemoglobina fetal abaixo de $10 \mathrm{~g} / \mathrm{dL}$, sendo importante marcador não invasivo de anemia fetal.

Nossos resultados não permitem ainda abandonar os procedimentos invasivos no reconhecimento da anemia fetal, mas sugerem que o PVS na dopplervelocimetria da ACM pode ser utilizado como método auxiliar no diagnóstico não invasivo da anemia fetal e no acompanhamento pré-natal do feto anêmico submetido ao tratamento intra-uterino.

A meta de desenvolver-se método não invasivo de alta sensibilidade é poder promover a redução dos métodos invasivos que só seriam utilizados após seleção prévia.

\section{ABSTRACT}

Purpose: to assess the correlation between middle cerebral artery peak systolic velocity and umbilical cord blood hemoglobin concentration and to determine its diagnostic value.

Patients and Methods: a cross-sectional prospective study was performed from January 2000 to May 2003. Forty-four isoimmunized pregnant women underwent a protocol for the identification of fetal hemolysis. When intrauterine transfusions were indicated, the umbilical cord blood hemoglobin concentration was measured at the beginning of the procedure. Each intrauterine transfusion preceded by Doppler velocimetry of the middle cerebral artery was regarded as one case, summing up eighty-three procedures. In all cases, the middle cerebral artery Doppler examinations were performed within the three hours preceding fetal blood sample collection. The systolic velocity peak was recorded and considered abnormal when its value was above 1.5 times the median for the corresponding gestational age. Hemocue ${ }^{\circledR}$ (B-Hemoglobin Photometer Hemocue AB; Angelholm, Sweden) was the device used to measure fetal hemoglobin 
concentration. The relationship between middle cerebral artery peak systolic velocity and cord blood hemoglobin was obtained by the $\chi^{2}$ test, considered significant at $p<0.05$. Results: in thirty-three cases the cord blood hemoglobin concentration was below $10.0 \mathrm{~g} / \mathrm{dL}$. There was a strong correlation between the two measured variables $(p<0.001)$. The middle cerebral artery peak systolic velocity with values above 1.5 times the median was associated with cord blood hemoglobin concentration below $10 \mathrm{~g} / \mathrm{mL}(p<0,001)$. The sensitivity of an increased middle cerebral artery peak systolic velocity was $75.8 \%$ for the detection of a cord blood hemoglobin level of $10 \mathrm{~g} / \mathrm{dL}$ or lower.

Conclusion: the middle cerebral artery peak systolic velocity can be used as a noninvasive method for the diagnosis of fetal anemia.

KEYWORDS: High risk pregnancy. Fetus-maternal transfusion. Fetal anemia.

\section{Referências}

1. Cabral ACV, Pereira AK, Assreuy SS, Taveira MR. Isoimunização materna e doença hemolítica perinatal. Realidade e perspectivas. J Bras Ginecol 1998; 108:181-5.

2. Bowman JM, Pollock JM, Peterson LE, Harman CR, Manning FA, Menticoglou SM. Fetomaternal hemorrhage following funipuncture: increase in severity of maternal red-cell alloimmunization. Obstet Gynecol 1994; 84:839-43.

3. Bahado-Singh $\mathrm{R}, \mathrm{Oz} \mathrm{U}$, Deren $\mathrm{O}$, et al. A new splenic artery Doppler velocimetric index for prediction of severe fetal anemia associated with Rh alloimmunization. Am J Obstet Gynecol 1999; 180:49-54.

4. Nicolaides KH, Bilardo CM, Campbell S. Prediction of fetal anemia by measurement of the mean blood velocity in the fetal aorta. Am J Obstet Gynecol 1990; 162:209-12.

5. Taveira MR, Cabral ACV, Leite HV, Brum AP, Demian AA, Melo IG. Avaliação da velocidade média na aorta torácica descendente em fetos com anemia. Rev Bras Ginecol Obstet 2001; 23:653-7.

6. Mari G, Deter RL, Carpenter RL, et al. Noninvasive diagnosis by Doppler ultrasonography of fetal anemia due to maternal red-cell alloimmunization. N Engl J Med 2000; 342:9-14.

7. Vyas S, Nicolaides KH, Campbell S. Doppler examination of the middle cerebral artery in anemic fetuses. Am J Obstet Gynecol 1990; 162:1066-8.

8. Abdel-Fattah SA, Soothill PW, Carroll SG, Kyle PM. Noninvasive diagnosis in anemia in hydrops fetalis with the use of middle cerebral artery Doppler velocity. Am J Obstet Gynecol 2001; 185:1411-5.
9. Detti L, Mari G, Akiyama M, et al. Longitudinal assessment of the middle cerebral artery peak systolic velocity in healthy and in fetuses at risk for anemia. Am J Obstet Gynecol 2002; 187:937-9.

10.Stefos T, Cosmi E, Detti L, Mari G. Correction of fetal anemia on the middle cerebral artery peak systolic velocity. Obstet Gynecol 2002; 99:211-5.

11.Nishie EN, Brizot ML, Liao AW, Carvalho MH, Toma O, Zugaib M. A comparison between middle cerebral artery peak systolic velocity and amniotic fluid optical density at $450 \mathrm{~nm}$ in the prediction of fetal anemia. Am J Obstet Gynecol 2003; 188:214-9.

12.Kowalczyk D, Guzikowski W, Kubicki J, Maczka M. Doppler measurement of blood flow in the middle cerebral artery for the diagnosis of fetal anemia. Ginekol Pol 2003; 74:1160-5.

13.Pereira L, Jenkins TM, Berghella V. Conventional management of maternal red cell alloimmunization compared with management by Doppler assessment of middle cerebral artery peak systolic velocity. Am J Obstet Gynecol 2003; 189:1002-6.

14.Segata M, Mari G. Fetal anemia: new technologies. Curr Opin Obstet Gynecol 2004; 16:153-8.

15.Cabral ACV. Isoimunização materna. In: Cabral ACV, editor. Obstetrícia. $2^{a}$ ed. Rio de Janeiro: Revinter; 2002. p. 203-9.

16.Daffos F, Cappela-Pavlovsky M, Forestier F. Fetal blood sampling during pregnancy with use of a needle guided by ultrasound: a study of 606 consecutive cases. Am J Obstet Gynecol 1985; 153:655-60.

17.Weiner CP, Williamson RA, Wenstrom KD, et al. Management of fetal hemolytic disease by cordocentesis. II. Outcome and treatment. Am J Obstet Gynecol 1991; 165:1302-7.

18.Bernaschek G, Yildiz A, Kolankaya A, Stuempflen I, Deutinger J. Complications of cordocentesis in highrisk pregnancies: effects on fetal loss or preterm delivery. Prenat Diagn 1995; 15:995-1000.

19.Deren O, Önderoglu L. The value of middle cerebral artery systolic velocity for initial and subsequent management in fetal anemia. Eur $\mathrm{J}$ Obstet Gynecol Reprod Biol 2002; 101:26-30.

20.Mari G, Moise KJ Jr, Deter RL, Kirshon B, Stefos T, Carpenter RJ Jr. Flow velocity waveforms of the vascular system in the anemic fetus before and after intravascular transfusion for severe red-blood cell alloimmunization. Am J Obstet Gynecol 1990; 162:1060-4.

21.Copel JA, Grannum PA, Green JJ, et al. Fetal cardiac output in the isoimmunized pregnancy: a Doppler-echocardiographic study of patients undergoing intravascular intrauterine transfusion. Am J Obstet Gynecol 1989; 161:361-5.

Recebido em: 6/10/2003 Aceito com modificações em: 8/9/2004 